\title{
Diabetic Foot Exercise, and Care to Prevent Mortality and Morbidity in Diabetes Mellitus Patients in Pandemic Covid-19 Era: Case Report
}

\author{
Yuningtyaswari ${ }^{1}$, Titiek Hidayati ${ }^{2, *}$, Ayunindya Adila $^{3}$
}

\author{
${ }^{I}$ Department of histology, Faculty of medicine and health sciences, Universitas Muhammadiyah Yogyakarta.; \\ yuningtyas@umy.ac.id \\ ${ }^{2}$ Department of Epidemiology, Family Medicine and Public Health, Faculty of Medicine and Health Sciences, Universitas \\ Muhammadiyah Yogyakarta. \\ ${ }^{3}$ Faculty of medicine and health sciences, Univeritas Muhammadiyah Yogyakarta. \\ *Corresponding author. Email: hidayatifkumy@yahoo.co.id
}

\begin{abstract}
Non-pharmacological interventions that can be undertaken include a proper nutritional diet, regular physical exercise, and smoking cessation. Diet and regular exercise will change the glucose level of Type II Diabetes Mellitus sufferers to achieve standard metabolic control, normal HbA1c levels, and reduce vascular complications in Type II Diabetes Mellitus patients. The patient had a history of Diabetes Mellitus for approximately five years ago. Patients routinely go to Level 1 health facilities and routinely take medication every day.
\end{abstract}

Keywords: Diabetic Foot Exercise, Non-pharmacological Care, Pandemic Covid-19 Era

\section{INTRODUCTION}

Diabetes is a global health problem that needs to be handled carefully. The proportion of diabetes mellitus type II patients in Asia increased in a short time in Asia. According to WHO, the number of sufferers in Indonesia is estimated to have risen from 8.4 million in 2020 to 21.3 in 2030 [1].

Diabetes Mellitus type II (T2DM) is caused by metabolic disorders and is often the result of excess calorie intake compared to energy expenditure. A progressive defect in insulin secretion characterizes insulin resistance, increasing the body's insulin demand to maintain glucose homeostasis. Pancreatic $\beta$ cells that fail to release enough insulin to compensate for the increased need for insulin, blood glucose levels will gradually increase [2]

Chronic hyperglycemia causes clinical problems such as dysfunction, organ failure. The eyes, kidneys, nerves, heart, and blood vessels experience these clinical problems, which will increase the morbidity and mortality rate. [2].

Plasma glucose parameters underlie the diagnosis of diabetes, namely the fasting plasma glucose (FPG) test for plasma glucose levels $\geq 126 \mathrm{mg} / \mathrm{dL}$ or $7.0 \mathrm{mmol} / \mathrm{L}$, and the Oral Glucose Tolerance Test (OGTT). Patients are diagnosed with diabetes when the plasma glucose level is $\geq$ $200 \mathrm{mg} / \mathrm{dL}$ or $11.1 \mathrm{mmol} / \mathrm{L}$. Unhealthy lifestyles such as reduced activity and changes in bad diet habits will increase the percentage of the population that is overweight and obese [3].

Non-pharmacological interventions that can be undertaken include a proper nutritional diet, regular physical exercise, and smoking cessation. Glucose levels in obese type II diabetes mellitus patients will decrease by getting used to a healthy diet and regular exercise from moderate to intense. [3].

The combination of a healthy lifestyle and drug therapy is an effort to achieve normal metabolic conditions, normal HbA1c levels, decrease the risk of macrovascular complications for diabetes mellitus type II patients [4]. Diabetes treatment is available in an oral or injectable form, so that doctors must understand the types of most effective, safe, and well-tolerated drugs by the body. Metformin is still the first treatment option for most patients [4].

\section{CASE PRESENTATION}

The male patient aged 50 years presents with back and forth headaches since one week ago, pain sometimes radiating to the right and left shoulders. Complaints of pain worsen when used for strenuous activities, and complaints disappear when used to lie down. The patient rated the pain scale as a score of 3 . The patient was a little worried if the disease worsened with the appearance of this complaint. Patients can still carry out daily activities such as cooking, washing dishes, repairing damaged furniture, etc. Patients sometimes complain that his body is a bit itchy at night, and they sweat even though they don't have any activity. Complaints were not accompanied by chest pain, cold sweat, tightness, and swollen feet. There are no complaints about defecation and stool.

The patient had a history of Diabetes Mellitus for approximately five years. Patients routinely go to Level 1 health facilities and routinely take medication every day. 
History of hypertension, stroke, heart, and kidney disease is denied. The patient's mother had a history of diabetes mellitus, a history of hypertension, stroke, heart disease, and allergies in the family was dismissed.

Patients work as entrepreneurs; he feels enjoy and have no problems in their work. The patient is married and has two daughters. The patient has a good diet, three times a day, using brown rice or corn instead of white rice, always eating vegetables complete with side dishes boiled or steamed. Patients severely limit their consumption of sugary drinks and drink water more often. During the day, the patient takes a break at 12.00 WIB for prayer and lunch. During the daytime rest, the patient usually spends time taking a nap or watching television, and at night, starting at 21.30 WIB. Since being diagnosed with Type II Diabetes Mellitus, the patient has stopped smoking and is diligent in exercising three times a week.

The patient has accepted the disease and knows the risk factors and complications of the disease. The patient also understands that he must take medication for life to maintain his health. The patient always hopes that his life's quality is still good. Still, the patient thinks that type II Diabetes Mellitus can worsen even though the patient has taken medication regularly and maintains his lifestyle. Currently, the patient is worried about the disease. For example, the patient is only sick with the flu and is afraid of contracting various diseases, especially during the COVID19 pandemic. For example, the patient's hands and feet suddenly feel numb. Then the patient worries that the condition is worsening and complications of diabetic neuropathy occur. Patients sometimes tell their concerns to their families, and patients are quite satisfied with the family's response to their worries.

The cognitive and emotional dimensions of disease perception were recognized as influencing health, occupational involvement, and disease severity. The mental aspect includes the causes and course of the disease. Emotional conditions, such as levels of anger, fear, or mood swings, will affect health. The instrument used was the Brief Illness Perception Questionnaire (BIPQ). BIPQ consists of 9 questions. The first five numbers assess the patient's cognitive perceptions such as how strong the disease's influence is on daily life, patient beliefs about the chronic period of the disease, ability to control disease, patient confidence in disease treatment, and experience of symptoms experienced by the patient. Questions 6 and 8 serve to assess the patient's feelings and emotional response to illness. Question number 7 sets the patient's level of knowledge of the disease. The last question is an open question in the form of 3 things according to the patient that causes a disease. The maximum total score on the questionnaire is 80 , and the minimum score is 0 . In patients, the total score obtained is 55 points. Research by Løchting (2013)[5] states that a higher score indicates that the disease is considered a threat.

The family's functional level was assessed using the APGAR score instrument, which consisted of 5 questions. This score shows the level of patient satisfaction and sees whether there is family dysfunction or not. The family is moderately dysfunctional if an APGAR score of 4-7 is obtained, and an APGAR score is 0-3 for severe dysfunction. The patient got an APGAR score of 9, which means that the patient's family is very functional. The patient lives at home with his wife and two daughters, and the patient's relationship with his wife and two daughters are good. The patient is less active in recitation activities or community meetings. The condition of the patient's home environment is quite good. There is a yard, adequate ventilation, and is not close to other houses, but the patient's place is quite crowded because it is located on the highway's side. The patient's home has two floors, walls, tiled floors, with a tile roof. Water sources from wells and water reservoirs are located beside the house. Clean and healthy living behavior in the patient's household is assessed by the indicator of Clean and Healthy Living Behavior, and the patient's family has implemented behavior, which consists of 10 indicators.

Researchers began to create instruments to assess overall well-being, namely physical, mental, and social well-being. This concept will later be known as HealthRelated Quality of Life (HRQoL). HRQoL assessment uses special instruments such as the Short Form 36 (SF-36), which has been used in various countries and has become the gold standard in assessing life quality. This instrument contains 36 short questions covering eight aspects: physical characteristics, emotional elements, social factors, physical health aspects, emotional health aspects, pain aspects, fatigue aspects, and general health aspects [6,7]. They measured the quality of life using a questionnaire sheet with four objective criteria, namely the excellent quality of life (76-100), good quality of life (51-75), adequate quality of life (26-50), low quality of life (0-25). The patient gets a score of 76 , which means that the quality of life is excellent [7].

Examination of vital signs BP: $120 / 90 \mathrm{mmHg}$, RR: $12 \mathrm{x}$ / minute, Pulse: 73, x / minute, Temperature: 36.8 oC. Anthopometric examination of TB $173 \mathrm{~cm}$, weight $75 \mathrm{~kg}$, so that a BMI of 25.08 was obtained, including grade I obesity criteria. Physical examination, Thorax: no chest wall retraction, sonor, vesicular baseline sound, etc. were within normal limits but found hypesthesia. in the lower left limb. Laboratory results: GDP $113 \mathrm{mg} / \mathrm{dL}(<100 \mathrm{mg} / \mathrm{dL})$, HbA1c: $7.6 \%$ (4\% -5.6\%), Total cholesterol $239 \mathrm{mg} / \mathrm{dL}$ (<200 mg / dL), Triglycerides $180 \mathrm{mg} / \mathrm{dL}(<150 \mathrm{mg} / \mathrm{dL})$, HDL $65 \mathrm{mg} / \mathrm{dL}(>40 \mathrm{mg} / \mathrm{dL})$, and LDL $119 \mathrm{mg} / \mathrm{Dl}$ (<100 mg / dL). Suggestion: additional examination in the form of AP / Lateral chest radiograph and Ankle Brachial Index (ABI) measurement.

The holistic diagnosis is cephalgia, grade I obesity, dyslipidemia, and type II diabetes mellitus in a middle-aged male with a less healthy and healthy lifestyle. After receiving education, this patient who works as an entrepreneur begins to understand the disease and has the awareness for regular control at the first level of health facilities - patients' understanding of using drugs, doing physical activity, and having a healthy lifestyle increased. Pharmacological therapy given to this patient was 
Metformin 2x500mg, Glimepirid 1x2mg, and Pioglitazone $\mathrm{HCl} 1 \times 30 \mathrm{mg}$. Patients were given three kinds of antidiabetic medicines, i.e., the biguanide, thiazolidinedione, and sulphonylurea groups.

Non-pharmacological therapies applied to a healthy lifestyle include calculating daily calorie needs, following a nutritious diet, and controlling the recommended food composition: carbohydrates $45-65 \%$ of total energy fat 20 $25 \%$ of total intake. Energy and protein $10-20 \%$ of total energy intake. Not only that, but patients can also maintain regular physical activity, perform independent foot care, and do diabetic foot exercises.

Diabetes patients can improve their quality of life by preventing foot ulcers through foot care, another way of motivating themselves to control diabetes complications [8]. If these efforts are not made so that complications occur, the diabetic foot will significantly impact life quality. The impact is decreased mobility and possible foot injury. The above conditions affect the patient's ability to perform simple daily tasks. [9].

Foot care that can be done by patients are:[10] 1) No walking barefoot 2) Always check the feet every day for redness or wounds 3) keep the feet clean, not wet and apply a moisturizing cream to the skin dry feet, 4) cut nails regularly 5) use cotton socks 6) shoes should not be too narrow or loose and do not use high heels.

Foot exercise is carried out for 15-20 minutes to make the blood supply from the heart channeled through the femoral artery leading to the popliteal and dorsalis pedis $[11,12]$. Plantar flexion, dorsiflexion, inversion, eversion, and toe circumcision are foot exercises (Figure 1). Activities performed after taking insulin should include exercises 5 to 10 with 10 to repeat. Patients should stop exercising if they feel nauseous, dizzy, or tired [13].

Foot exercises that can be done as follows:

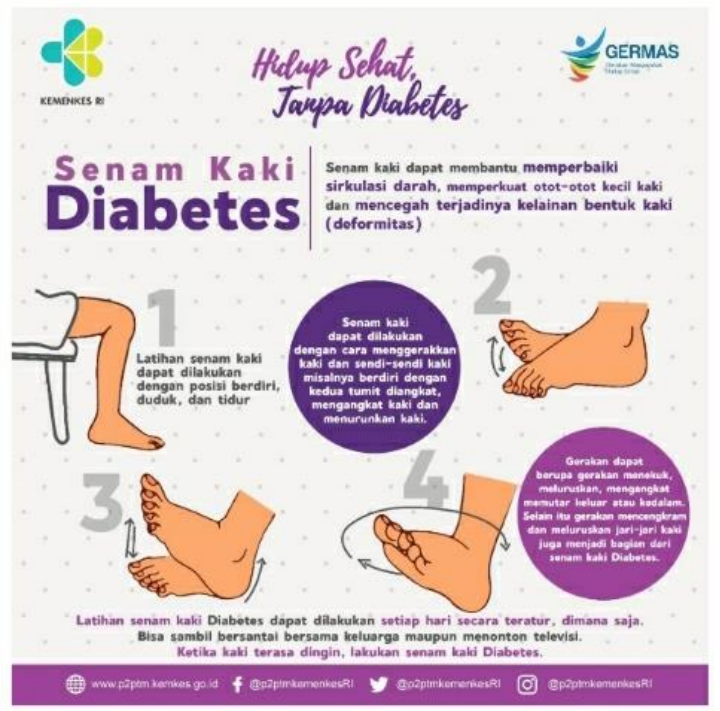

Figure 1.Diabetic Foot Exercise Movement (1) Outcome and Follow Up

On $(23 / 6)$, the patient first learned to calculate daily calorie needs and know the types of foods with a low glycemic index. There are no difficulties during the learning and education process regarding caloric and nutritional requirements because patients often ask doctors at firstlevel health facilities. Education was continued by briefly explaining Diabetes Mellitus type II. We did not only explain to patients but also their families. We had discussions to resolve patients' concerns about their illness, and we needed family support to help improve the patient's Quality of Life.

ABI pressure was measured after education to the patient by measuring the systole pressure in both arms, and then the largest systole size was taken, $120 \mathrm{mmHg}$. Then, the systole pressure was measured in both legs of the patient, and the ABI pressure was obtained in the patient's right leg $(130 \mathrm{mmHg} / 120 \mathrm{mmHg}=1.08)$ and the left leg $(120 \mathrm{mmHg} / 120 \mathrm{mmHg}=1.00)$. Both limbs are still within the standard ABI limit, namely 1.00 to 1.30 .

The next day (24/6), the patient learns the correct diabetic foot exercises and will try to apply them three times a week. Patients are straightforward to grasp information because diabetic foot exercises are not too difficult. Furthermore, I educate foot care, and patients have been implementing it for the last two years. Patients say their feet are more comfortable with attention to foot health than when they have not paid attention to foot health.

The degree of overall well-being, namely physical, mental, and social well-being (Health-Related Quality of Life), is monitored using special instruments such as Short Form 36 (SF-36). SF-36 consists of 36 short questions covering eight aspects: physical aspects, emotional aspects, social aspects, physical health aspects, emotional health aspects, pain aspects, fatigue aspects, and general health aspects. The score (22/6) was 76 (excellent quality of life). The increase in score occurred after education and monitoring for one week. The score (26/6) was 84 , although the interpretation was the same; namely, the quality of life was excellent.

Patients' cognitive and pain perception of their disease were assessed using The Brief Illness Perception Questionnaire (BIPQ). The BIPQ consists of 9 questions with the maximum total score on the questionnaire is 80 and the minimum score is 0 . A higher score indicates that the disease is considered a threat by the patient. The score $(22 / 6)$ is 55 , which means that type II DM is regarded as a sufficient threat by the patient. The increase in score occurred after education and resolution of patient concerns. The score was (26/6), namely 63.

The APGAR score increases after the patient is educated about calorie counting, the patient's nutrition, and knowledge about type II Diabetes Mellitus and the family's critical role in increasing the patient's QoL. The APGAR score on $(22 / 6)$ is nine, and one week later, the APGAR score becomes 10 , which means that the family is very functional, and the score increases in the way the patient's family discusses things with the patient and shares problems with him. 
The patient started to apply foot exercises at night (24/6) after the education and used it until the date (26/6). At the end of the week, the ABI pressure was checked, the ABI pressure was obtained in the patient's right leg $(137 \mathrm{mmHg}$ / $125 \mathrm{mmHg}=1.1)$ and the left leg $(132 \mathrm{mmHg} / 125 \mathrm{mmHg}$ $=1.056)$. ABI pressure experienced a difference after the patient did regular diabetic foot exercises.

\section{RESULT AND DISCUSSION}

Diabetes mellitus (DM) is a metabolic disease characterized by chronic hyperglycemia resulting from insulin secretion changes or action (Kaur and Kochar, 2017). Hyperglycemia is a medical condition in the form of an increase in glucose levels in the blood beyond normal limits[10].

A problematic treatment plan is determining what to eat and following a meal plan. The meal plan should adapt to individual needs or no one-size-fits-all diet for diabetics [13] [14]. All patients received individualized nutritional therapy by a dietitian [14]. Healthy therapy methods generally use basic visual food planning guidelines that are role-playing or show calorie control methods such as displaying smaller plates and examples of low-carbohydrate ingredients [15].

The glycemic index (GI) is a critical concept proposed in choosing suitable foods for people with diabetes. GI is a measure of how food increases blood glucose levels after consumption (Nuzul et al., 2016). Low GI values are below 55 , moderate GI values are between 55 to 69 , and high GI values are above 70 [16].

The glucose level in carbohydrate food sources such as grains, tubers, fruits, and nuts varies depending on the type of ingredient. Food ingredients with a lower glycemic index than white rice are the right alternative for people with diabetes. The low glycemic index group elements were ambon bananas, boiled red beans, sorghum rice, steamed kepok bananas, brown rice, ripe green beans, and corn, while the medium group was steamed breadfruit. Boiled cassava and steamed bentul are still classified as foods with a high GI, so it is recommended for people with Type II DM to look for other tubers with low glycemic index [17].

Studies show that a low GI diet has benefits in reducing HbA1c (P-value <0.0001), fasting blood sugar $(\mathrm{P}$-value $=$ $0.01)$, BMI $(\mathrm{P}$-value $=0.01)$, and blood lipids $(\mathrm{P}$-value $=$ 0.003) [18]. The improvements in fasting blood sugar and HbA1c suggest a possible increase in insulin sensitivity and a significant achievement for dietary interventions that can reduce weight in people with prediabetes or diabetes during a certain period [18].

Long-term consumption of high GI foods is proposed to increase insulin demand, improve insulin resistance, impair pancreatic $\beta$ cell function, and ultimately lead to type II Diabetes Mellitus [19]. Other studies have also linked reduced incidence and prevalence of heart disease, diabetes, and some cancer forms with long-term low-GI foods consumption [20].
The patient's knowledge of diabetes will form the basis when the patient makes decisions about diet, exercise, weight control, blood glucose monitoring, eye and foot care to prevent complications. Diabetic foot injury is one of the chronic complications of diabetes Mellitus type II, which is the worst result of its management. If your diabetic foot is not adequately cared for, it can easily become injured and quickly develop into gangrenous ulcers if not treated properly. Diabetes Mellitus patients with complications of the diabetic foot have a significant impact on the quality of life, for example, loss of mobility associated with foot injuries, which can affect the patient's ability to perform simple daily tasks [8].

Diabetic foot ulcers can be prevented by conducting early screening and diabetic foot care education for highrisk individuals [10]. The goals of diabetes foot care are to detect any abnormalities as early as possible, maintain foot cleanliness, and prevent foot injuries that can pose a risk of infection and amputation.

People with diabetes who have sufficient knowledge about diabetic foot care are the basis and motivation to control the disease's complications [8]. There is a significant relationship between foot knowledge and care in diabetic patients. Diabetes patients who have good experience with foot care are 4.7 times more likely to do foot care and notice changes in themselves, such as numbness of the feet, than those with less knowledge $(\mathrm{P}$-value $=0.024)$. This study is by the patient; the patient has sufficient experience to treat diabetic foot to make the patient more enthusiastic about preventing diabetic foot/ulcer complications.

The components of foot care recommended for people with diabetes [17,22 ] consist of 1) Washing and drying feet daily using mild soap and warm water. 2) Checking the feet' consistency every day to see the presence of dryness and cracks, blisters, sores, redness, warmth, and swelling when touched. There are ingrown toenails, calluses, and calluses. 3) Caring for nails. Nail cutting is recommended after bathing when the condition of the pins is still soft. Nails should be cut using nail clippers, cut horizontally, and no corners on the nails. 4) Be careful when exercising. People with diabetes are advised not to walk barefoot and wear comfortable shoes while exercising. 5) Protect the feet with shoes and socks. 6) Maintaining smooth blood flow to the legs, elevating the legs when sitting, moving the fingers and leg joints, or doing diabetic foot exercises.

Physical activity and exercise therapy aim to prevent risk factors for foot ulcers [23] and help promote wound healing [13]. The patient may experience thickening, stiffness of the main tendons and ligaments of the ankle, namely the plantar fascia and Achilles tendon. This condition can develop into inflammation, microvascular disease, and trauma if used excessively. Foot gymnastics is an exercise performed for people with Diabetes Mellitus (DM) or non-sufferers to prevent injuries and help blood circulation in the legs [21]

A study on the effectiveness of leg exercises for 12 weeks found that in the group of diabetes mellitus type II sufferers, there was an increase in muscle function in the 
flexor digitorum brevis $(25.0 \%)$ and tibialis anterior $(25.0 \%)$, indicating that exercise can slow the prognosis of chronic complications of diabetes [22]. The authors reported a significant reduction in the pressure drop in the heel of $1.7 \%(\mathrm{P}$-value $=0.03)$ and the lateral forefoot by $2.1 \%(\mathrm{P}$-value $=0.01)$, as well as a significant reduction in pressure in the midfoot $(23,3 \%)$, $\mathrm{P}$-value $<0.01)$ and on the lateral forefoot $(8.8 \%, \mathrm{P}$-value $<0.01)$ [22].

The patient's quality of life was assessed using the SF36 instrument. Based on these instruments, patients who routinely exercise diabetes get a better quality of life score, namely physical function score $(7.0 \%, \mathrm{P}$ value $=0.028)$, body pain $(17.6 \%, \mathrm{P}$ value $=0.009)$, limited physical role. $(29.0 \%), \mathrm{P}$ value $=0.006)$, emotional role limitations $(30.3 \%, \mathrm{P}$ value $=0.002)$, and social function $(15.6 \%, \mathrm{P}$ value $=0.001)[22]$.

This systematic review's main finding is that diabetic exercise is a non-pharmacological treatment that is beneficial in the outcome of the diabetic foot, especially in increasing the conduction velocity of nerves in the lower limbs. Increased skin sensitivity and intraepidermal nerve fiber density can delay the occurrence of diabetic peripheral neuropathy, skin damage, and ulceration because the lack of sharpness will cause a lack of oxygen supply so that bacteria can reproduce, especially anaerobic bacteria [10]. According to research conducted by Mirtha et al. (2018), a diabetic exercise carried out for at least 12 weeks is not only to prevent foot ulceration but also to reduce the area of ulceration $(\mathrm{P}$-value $=0,000)$. Diabetic foot exercise performed by the elderly three times a week for four weeks can also increase foot sensitivity $(\mathrm{P}$-value $=0,000)$ and reduce blood sugar levels in people with Diabetes Mellitus type II in the elderly $(\mathrm{P}$-value $=0,000)[13]$.

Diabetes mellitus sufferers who experience impaired sensitivity due to impaired blood flow can be assessed by measuring the Ankle Brachial Index (ABI). A low ABI can be interpreted as a decrease in sensitivity and ischemia, starting from mild, moderate, to severe ischemia [10].

Diabetic exercise increases the Brachial Index pressure significantly in diabetic patients who have done foot exercises four times a week $(\mathrm{P}$-value $=0.046)[11]$. Diabetes exercise can also reduce blood sugar levels since the first treatment $(\mathrm{P}$-value $<0.05)$, this is because the effect of these leg exercises can increase cell sensitivity to insulin so that blood sugar will enter cells for metabolic processes [12].

Another research was conducted with the One group pretest-postest design approach. The population was all patients with Type II Diabetes Mellitus is one of the public health center areas in Payakumbuh City, with as many as 77 people. Based on the study results, it is known that diabetes exercise can increase ankle-brachial index pressure $(\mathrm{P}$ value $=0.005)$ and can increase peripheral perfusion and prevent complications of Type II Diabetes Mellitus [12].

On the first day of the patient's ABI, the pressure was measured, and it was found that the right leg was 1.08, and the left leg was 1.00. After one week of doing diabetic foot exercises, the ABI pressure in both legs increased. The right leg increased to 1.10 , and the left leg increased to 1.056

In doing foot exercises for 15-20 minutes, the leg muscle cells need energy in the form of a blood supply from the heart channeled through the femoral artery leading to the popliteal and dorsalis pedis. This situation is seen with an increase in pulse after the foot exercise treatment $4-10$ times/minute and an increase in systolic pressure of $5-10$ $\mathrm{mmHg}$. This condition indicates that the heart as a central circulation has provided additional energy for the muscle cells used during leg exercises. [12,13].

The leg exercises' movements are as follows [24] The patient sits upright on a bench with his feet touching the floor. 2) With the heel resting on the floor, the toes of both feet are straightened up and then bent back down like a chicken claw ten times. 3) Putting the heel of one foot on the floor, lift the sole up. On the other hand, the toes are placed on the other leg's bottom, and the feet' heels are lifted. This movement is carried out simultaneously on the right and left legs alternately and is repeated ten times. 4) The heel of the foot is placed on the floor. Then lift the tips of the toes and make a circular motion on the ankles ten times. 5) Toes are placed on the floor. Then raise the heel and make a circular motion by moving the ankle ten times. 6) Then lift one knee of the leg, and straighten it. Then move your toes forward, then lower them back alternately left and right. Repeat this movement ten times. 7) Next, straighten one leg on the floor, lift that leg, move the tip of your toes towards your face, and lower it back to the floor. 8) Raise both legs and then straighten them. Repeat the same as in step 8, but use both right and left legs simultaneously. Repeat this movement ten times. 9) Raise both legs and straighten them to maintain that position. Then move the ankle back and forth. 10) Then straighten one leg, lift it, rotate the foot on the ankle, and do a movement like writing in the air with the legs from numbers 0 to 10 do alternately. 11) Put a sheet of newspaper on the floor. Then the newspaper shape becomes like a ball with both legs. Then open the ball back into the sheet as before using both legs. This movement is done only once. 12) Then, tear the newspaper into two parts, then separate the newspaper's two sections. 13) Some of the newspaper was torn into small pieces with both legs. 14) then transfer the collection of torn pieces with both legs and place the torn paper on the whole amount of paper earlier. 15) Then, wrap all the parts with both right and left legs into a ball.

\subsection{Patient Perception}

During the diabetic leg exercises, I had no trouble because the movements are simple and can be done while sitting. I have been exercising so that doing diabetic foot exercises is not tricky. I became accustomed to doing diabetic exercises in the morning because I felt a difference after doing leg exercises, namely that my left leg was less numb. I hope to do this exercise regularly, at least four times a week, to increase my feet' sensitivity and reduce the numbness lately. 
After I could count calories and get information about nutritional therapy, I felt that my body was more comfortable. I didn't tire because there wasn't too much food intake and enough to meet my daily needs. I hope that I can lose weight. I am a little overweight, so I want to stabilize my weight after losing a little to prevent obesity and heart disease. I do routine foot care, and I hope that regular foot care can prevent my feet from getting injured

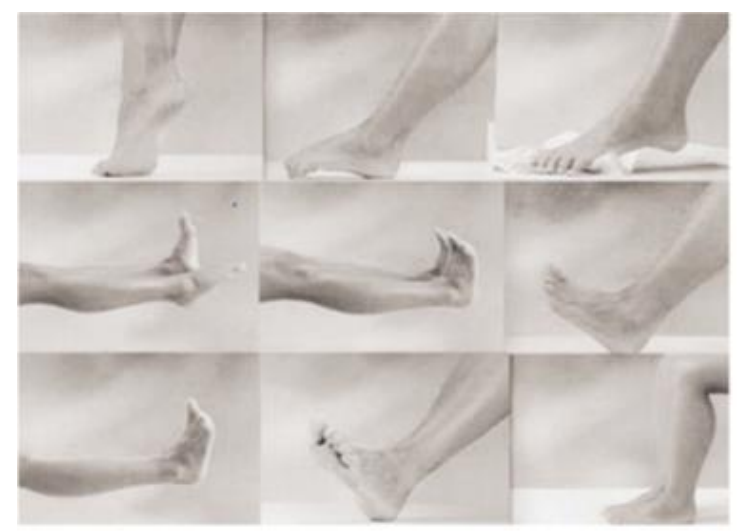

Figure 2. Diabetic Foot Exercise Movement.

\section{CONCLUSION}

From the results of monitoring the low glycaemic index nutritional therapy program, diabetic foot care, and diabetic exercise in a type II Diabetes Mellitus sufferer, by assessing the quality of life and education regarding PHBS behavior in the household and not smoking in the home, the conclusions are:

1. Food ingredients with a lower Glycemic Index than white rice can be used as an alternative material for people with type II diabetes. Materials with low glycemic index are ambon bananas, boiled red beans, sorghum rice, steamed kepok bananas, brown rice, ripe green beans, and corn. At the same time, steamed breadfruit is a medium category ingredient.

2. A low GI diet has benefits in reducing HbA1c, fasting blood sugar, BMI, and blood lipids. The improvements were seen in fasting blood sugar, and HbA1c suggest a possible increase in insulin sensitivity.

3. Diabetic foot ulcers are one of the chronic complications of diabetes Mellitus type II, which is the worst result of its management. Diabetic foot ulcers can be prevented by conducting early screening and diabetic foot care education for high-risk individuals.

4. The goals of diabetes foot care are to detect any abnormalities as early as possible, maintain foot cleanliness, and prevent foot injuries that can pose a risk of infection and amputation.

5. Foot gymnastics is an exercise performed for people with Diabetes Mellitus to prevent injuries and help blood circulation in the legs.

6. Diabetic exercise, which is done to prevent foot ulceration and reduce the area of ulceration, can increase foot sensitivity, which is indicated by an increase in anklebrachial index pressure and a decrease in blood sugar levels for people with Diabetes Mellitus type II.

\section{AUTHORS' CONTRIBUTIONS}

$\mathrm{Y}$ and TH contribute to collecting data, and the second author/TH has contributions as analyze data.

\section{ACKNOWLEDGMENTS}

Acknowledgments of the research have given for Universitas Muhammadiyah Yogyakarta.

\section{REFERENCES}

[1] C. Y. Park., J. G. Kang., S. Chon., J. Noh., S. J. Oh., C. B. Lee., \& S. W. Park. 2014. Comparison between the Therapeutic Effect of Metformin, Glimepiride and Their Combination as an Add-On Treatment to Insulin Glargine in Uncontrolled Patients with Type 2 Diabetes. PLoS ONE, 9(3):87799

[2] S. Del Prato. Role of glucotoxicity and lipotoxicity in the pathophysiology of Type 2 diabetes mellitus and emerging treatment strategies. Diabet Med 2009;26: 1185-1192.

[3] Zhao, Yingzheng. et al.. Type 2 Diabetes MellitusDisease, Diagnosis, and Treatment. Journal of Diabetes \& Metabolism, 2015;06(05).

[4]. J. J. Marín-Peñalver., I. Martín-Timón., C. SevillanoCollantes., \& F. J. Cañizo-Gómez,. del. (2016). Update on the treatment of type 2 diabetes mellitus. World Journal of Diabetes, 7(17): 354 - 395..

[5] I. Løchting., A. M. Garratt., K. Storheim., E. L. Werner., M. Grotle. 2013. Evaluation of the Brief Illness Perception Questionnaire in Sub-Acute and Chronic Low Back Pain Patients: Data Quality, Reliability, and Validity. Journal Pain and Relief. 02(03).7

[6] L. Lins, \& F. M. Carvalho, 2016. SF-36 total score as a single measure of health-related quality of life: Scoping review. SAGE Open Medicine, 4: 1-12.

[7] Falah, Nabil Mubtadi. et al., 2017. Reliability and Validity Test of the Indonesian Language Short Form 12 Quality of Life Questionnaire in Rheumatoid Arthritis Patients. Indonesian Journal of Internal Medicine, 4(3), pp. 105 - 111

[8] Srimiyati. 2018. Knowledge of Diabetic Foot Prevention for Diabetes Mellitus Patients Affects Foot Care. MEDISAINS, 16(2), pp. 76-82.

[9]. S. Rohmah. 2019. Factors Affecting Behavior of Diabetic Foot Injury Prevention in Diabetes Patients. Midwifery Journal of Galuh University, 1(1), pp. 2336. 
[10] Perkeni. 2011. Consensus on the Management and Prevention of Type 2 Diabetes Mellitus in Indonesia 2011. PB. Perkeni: Jakarta.

[11] A. Santosa., W. Rusmono, 2016. Foot Exercise To Control Blood Sugar Levels And Lower Brachial Pressure In Diabetes Mellitus Patients. Scientific Journal of Health Sciences, 14(2), pp. 24 - 34

[12] A. Wahyun., N. Arisfa, 2016. Diabetic Foot Exercise Effectively Increases Ankle Brachial Index for Type 2 Diabetes Mellitus Patients. Journal of Applied Science and Technology, 9(2), pp. 155 - 164.

[13] L. T. Mirtha., M. Ariono., S. M. Putra. (, 2018). The Effect of Foot Exercise on Diabetic Patients with Foot Ulcer: An Evidence-Based Case Report. J Diabetes And Metabolics Disorder 5: 26.

[14] S. Wang., \& L. Copeland, (2015). Effect of Acid Hydrolysis on Starch Structure and Functionality: A Review. Critical Reviews in Food Science and Nutrition, 55(8), 1081-1097

[15]. M. A. Powers., J. Bardsley., M. Cypress, et al. Diabetes self-management education and support in type 2 diabetes: a joint position statement of the American Diabetes Association, the American Association of Diabetes Educators, and the Academy of Nutrition and Dietetics. Diabetes Care, 2015;38:1372,,-1382.

[16] W. D. Nuzul, et al., 2016. Evaluation of Glucose Content and Glycemic Index of Several Carbohydrate Sources in the Effort of Extracting Low Glycemic Index Food. Indonesian Journal of Pharmacy and Pharmaceutical Sciences, 3(2), pp. 67-73

[17].F. S. Atkinson., K. Foster-Powell., \& J. C. rand-Miller, 2008. International table of glycemic index and glycemic load values: 2008. Diabetes Care; 31; 228183.

[18] M. I. Zafar., et al. 2019. Low-glycemic index diets as an intervention for diabetes: a systematic review and meta-analysis. The American Journal of Clinical.00: 112

[19] C. S. Bobaru., D. Pasareanu. Giannakopoulou, Automated assume-guarantee reasoning by abstraction refinement, in A. Gupta, S. Malik (Eds.), Proceedings of the Computer Aided Verification, Springer, Berlin, Heidelberg, 2008, pp. 135-148. DOI: https://doi.org/10.1007/978-3-540-70545-1_14

[20] P.C.Banik, L. Barua, M. Moniruzzaman, R. Mondal, F. Zaman, F., \& L. Ali, L. (2020). risk of diabetic foot ulcer and its associated factors among Bangladeshi subjects: a multicentric cross-sectional study. $B M J$ Open, 10(2), e034058. doi:10.1136/bmjopen-2019034058

[21] F.S. Chiwanga., \& M. A. Njelekela. (, 2015). Diabetic foot: prevalence, knowledge, and foot self-care practices among diabetic patients in Dar es Salaam,
Tanzania - a cross-sectional study. Journal of Foot and Ankle Research, 8(1)

[22] I. Sejana. et al., 2018. Diabetes Mellitus Patient Behavior in Prevention of Diabetic Foot. Medika Health Journal Udayana, 4(1), pp. 38 - 46

[23] P. Francia., M. Gulisano., R. Anichini., \& G. Seghieri, 2014. Diabetic Foot and Exercise Therapy: Step by Step The Role of Rigid Posture and Biomechanics Treatment. Current Diabetes Reviews, 10(2), 86-99

[24] C.Diabetes., Pharmacologic Approaches to Glycemic Treatment: Standards of Medical Care in Diabetes2019. Diabetes Care, 42(Supplement 1), S90-S102. doi:10.2337/dc19-s009 